\title{
The Estimated Formation Pressure During Drilling Operations, in Drilling Wells, and an Exploration of Steps towards Efficient Drilling
}

\author{
Mahdi Imanian Najafabadi \\ Department of Automation and instrumentation \\ Petroleum University of Technology \\ Ahwaz , Iran
}

\begin{abstract}
Application of estimation and monitoring in drilling industry has found a wide-spread attention among researchers . One of the main areas relates to the efficient supervision and control of drilling operations . For instance, during drilling oil wells , a fluid is pumped into drill string . This fluid is circulated through drill pipe and drill bit to bottom of the well and then is directed to the surface via annulus to transfer cutting material, meanwhile, cooling and lubricating the drilling devices located at the bottom of well. This causes the pressure between the drilling fluid and formation to be varied , leading to kick phenomenon and ultimately resulting into probable blow out if it is left uncontrolled. Therefore, estimation and monitoring of bottom hole pressure is treated as a necessary requirement during well drilling operations [2] , [3].

In this paper, we are interested to investigate the feasibility of using adaptive observer technique to realize monitoring of drilling operations in oil wells from the essential and critical operational problem views, being considered in the work.

The resulting developed estimation and monitoring systems will be implemented and evaluated in simulation environment on the basis of accessible operational data from candidate oil wells.
\end{abstract}

\section{Keywords}

Estimation , Drilling Operation , Bottomhole Pressure , Adaptive Law , Lyapunov Law

\section{INTRODUCTION}

Due to the importance of the rotation system of mud drilling, it is recognized as the heart of the drilling operation. By importing hydrostatic pressure from drilling mud, the fluid formation is prevented from flowing from the wellbore to the surface. In other words, appropriate hydrostatic pressure created by drilling fluid prevents it from reaching to the kick off point and wellbore blow out. For creating the appropriate hydrostatic pressure, it is necessary to measure the pressure at the bottom of the formation continuously by sensors installed on the drilling bit. The pressure is sent to the surface, and the geologist decreases or increases the mud weight based on the pressure at the bottom of the wellbore. The base on the data acquisition and the choke valve is either closed or opened in order to provide appropriate pressure on the drilling bit .In some situations such as a pipe connection, the rotation system of mud is stopped, and the pipes are transferred from the wellbore to the surface. In reality there are no sensors to measure the pressure of the formation at that time, therefore it is possible to have sudden flow from the formation to the wellbore, and blow outs happen during the pipe connection operation. Therefore, pressure sensors are not applicable in this situation and cannot be used to monitor the formation pressure .In this study, we are looking for a solution to observe and measure the formation pressure during all drilling steps, including during the pipe connection. The problem is solvable by using the adaptive observer and estimator to estimate the pressure of the formation when the sensors are not inside the wellbore. Theses estimators and observers proposed by the lyapunov law are applicable in both automation and control engineering. After determining the mathematical relationships for the estimation of the formation pressure by an adaptive observer, the results provided from the adaptive observer and estimator are simulated to real data, and the acquired data are analyzed. [7] , [8] , [9] , [10]

\section{THE DESIGN OF THE ADAPTIVE OBSERVER AND ESTIMATOR}

For the estimation of formation pressure by the comparison estimator, it is necessary to develop a mathematical model from the drilling well in dynamic mode. Then, with the presented mathematical model, the lyapunov law is applied and the comparison raw for the observer is obtained.

\section{2-1. The mathematical model for the dynamic mode of the wellbore}

Model [1] has recently been used as one of the nonlinear mathematical models for solving the dynamic mode of wellbore. In the model, the drilling well is assumed to be isothermal, and the drilling fluid is the only liquid phase.

According to Figure 1, the drilling fluid is pumped by a mud pump and the fluid is injected through the drillstring to the bottom of the wellbore. After passing the fluid through the drilling bit and annular, it reaches the control choke valve. The proposed mathematical model classifies the drilling well into two parts, such as the drillstring and the annulus.

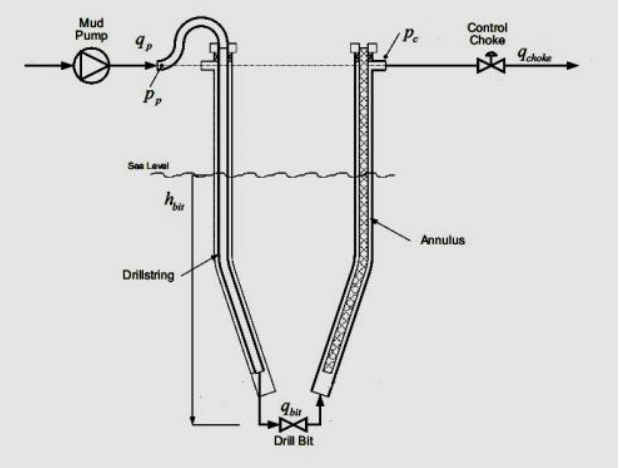

Figure 1 : Dynamic Model Schemes 
The final acquired equations of model [1] are presented as following [4] , [5] :

$\dot{x}_{1}=a_{1}\left(u_{1}-x_{3}\right)$

$\dot{x}_{2}=b_{1}\left(x_{3}+u_{2}-v_{1}\right)$

$\dot{x}_{3}=a_{2} x_{1}-a_{2} x_{2}-\theta_{1} x_{3}^{2}+\theta_{2} v_{2}$

$P_{b i t}=x_{2}+M_{a} \dot{x}_{3}+F_{a} x_{3}^{2}+\rho_{a} g v_{2}$

$b_{1}=\frac{\beta_{a}}{v_{a}}, \quad a_{2}=\frac{1}{M} \quad, \quad a_{1}=\frac{\beta_{d}}{v_{d}}, \quad x_{3}=q_{b i t}$,

$x_{2}=P_{c}, x_{1}=P_{p}, u_{1}=q_{\text {pump }}, u_{2}=q_{\text {chock }}$,

$v_{1}=\dot{v}_{a} \quad, v_{2}=h_{b i t} \quad, \quad \theta_{1}=\frac{F_{d}+F_{a}}{M}$,

$\theta_{2}=\frac{\rho_{d}-\rho_{a}}{M} g$

Where the above (corresponding) variables are defined as following:

$P_{p}$ : The input pressure of the drilling mud in the drillstring

$P_{c}:$ The output pressure of the drilling mud in the annulus

$M$ : Equivalent mass for the drilling mud in the drillstring

$B_{a}$ : The Bulk modules of the drilling mud in the annulus

$B_{a}$ : The bulk modules of the drilling mud in the drillstring

$v_{a}$ : The volume of the drilling mud in the annulus

$v_{d}$ : The volume of the drilling mud in the drillstring

$q_{\text {pump }}:$ The flow rate of the input drilling mud to wellbore

$q_{b i t}$ : The flow rate of the drilling mud on the drilling bit

$h_{b i t}$ : The depth of the drilling bit from the surface

$\rho_{a}$ : The density of the drilling mud in the annulus

$\rho_{d}$ : The density of the drilling mud in the drillstring

$F_{a}$ : The frictional coefficient between the drilling mud and the annulus

$F_{d}$ :The frictional coefficient between the drilling mud and the drillstring

\section{2-2. The nonlinear observer for the estimation of the formation pressure}

Equation (4) is used due to the presented nonlinear model and regarding the estimation of the flow rate of drilling mud on the drill bit and the continuous monitoring of formation pressure. It should be noted that the pressure formation is obtained indirectly according to Equation (4) and its relation to the flow rate of drilling mud on the bit, the input pressure of the mud in the drillstring, and the output pressure of the mud in the annulus. Therefore, $q_{\text {bit }}$ is considered as an impossible measurement of the system and the adaptive estimator is applied to estimate the output.

$y=x_{3}=q_{b i t}$

From the change of variable we have:

$\xi_{1}=x_{3}+l_{1} x_{1}$

$l_{1}$ is a positive constant gain .

From the converting of equation (6) to the estimated form $\hat{\xi}_{1}$, and deriving the error equation and the derivative of the equation we have:

$\hat{\xi}_{1}=\hat{x}_{3}+l_{1} x_{1}$

$e=\tilde{\xi}_{1}=\xi_{1}-\hat{\xi}_{1}=x_{3}+l_{1} x_{1}-\hat{x}_{3}-l_{1} x_{1}=\tilde{x}_{3}$

$\dot{e}=\dot{\tilde{x}}_{3}=\dot{\xi}_{1}-\dot{\hat{\xi}}_{1}$

Equations (1) and (3) are substituted in equation (9)

$$
\begin{aligned}
& \dot{\xi}_{1}=\dot{x}_{3}+l_{1} \dot{x}_{1}=-l_{1} x_{3}-\theta_{1} x_{3}^{2}+\theta_{2} v_{2}+x_{1}-x_{2}+l_{1} u_{1} \\
& \dot{\hat{\xi}}_{1}=\dot{\hat{x}}_{3}+l_{1} \dot{\hat{x}}_{1}=-l_{1} \hat{x}_{3}-\hat{\theta}_{1} \hat{x}_{3}^{2}+\hat{\theta}_{2} v_{2}+x_{1}-x_{2}+l_{1} u_{1} \\
& \dot{e}=-l_{1} \tilde{x}_{3}-\theta_{1} x_{3}^{2}+\hat{\theta}_{1} \hat{x}_{3}^{2}+\hat{\theta}_{1} \hat{x}_{3}^{2}-\hat{\theta}_{1} \hat{x}_{3}^{2}+\tilde{\theta}_{2} v_{2} \\
& \dot{e}=-l_{1} \tilde{x}_{3}-\theta_{1}\left(x_{3}^{2}-\hat{x}_{3}^{2}\right)-\tilde{\theta}_{1} x_{3}^{2}+\tilde{\theta}_{2} v_{2} \\
& \dot{e}=\dot{\tilde{x}}_{3}=-l_{1} \tilde{x}_{3}-\theta_{1}\left(x_{3}^{2}-\hat{x}_{3}^{2}\right)+\tilde{\theta}^{T} \phi \\
& \phi\left(\hat{x}_{3}, v_{3}\right)=\left[\begin{array}{c}
-\hat{x}_{3}^{2} \\
v_{2}
\end{array}\right], \text { and } \tilde{\theta}=\left[\begin{array}{c}
\tilde{\theta}_{1} \\
\tilde{\theta}_{2}
\end{array}\right]
\end{aligned}
$$

With writing the lyapunov law, the following equations are derived,

$$
\begin{aligned}
& U\left(\tilde{x}_{3}, \tilde{\theta}\right)=\frac{1}{2} \tilde{x}_{3}^{2}+\frac{1}{2} \tilde{\theta}^{T} \Gamma^{-1} \tilde{\theta} \\
& \dot{U}\left(\tilde{x}_{3}, \tilde{\theta}\right)=\frac{1}{2} \dot{\tilde{x}}_{3}^{2}+\frac{1}{2} \tilde{\theta}^{T} \Gamma^{-1} \dot{\tilde{\theta}}
\end{aligned}
$$

With the substitution of the equation (14) to equation (17) equation (18) is yielded,

$$
\dot{U}=-l_{1} \tilde{x}_{3}^{2}-\theta_{1}\left(x_{3}^{2}-\hat{x}_{3}^{2}\right) \tilde{x}_{3}+\tilde{\theta}^{T}\left(\tilde{x}_{3} \phi+\Gamma^{-1} \dot{\tilde{\theta}}\right)
$$

With considering the law below and it as a positive definite matrix we have:

$$
\begin{aligned}
& \dot{\tilde{\theta}}=-\tilde{x}_{3} \Gamma \phi \\
& f\left(x_{3}, \hat{x}_{3}\right)=\left(x_{3}^{2}-\hat{x}_{3}^{2}\right) \tilde{x}_{3} \\
& x_{3}-\hat{x}_{3}=0 \Rightarrow f=0 \\
& x_{3}-\hat{x}_{3} \neq 0 \Rightarrow f \succ 0
\end{aligned}
$$

The third part of equation (18) included the term, $\tilde{\theta}^{T}\left(\tilde{x}_{3} \phi+\Gamma^{-1} \dot{\tilde{\theta}}\right)$ that is canceled by condition (19). The 
second part of equation (18) included the term, $\theta_{1}\left(x_{3}^{2}-\hat{x}_{3}^{2}\right) \tilde{x}_{3}$ where condition (21) is canceled or becomes positive. Finally, with the consideration of $l_{1}$, which it is a positive definite matrix under the estimator matrix , equation (18) is converted to equation (22).

$$
\dot{U} \leq-l_{1} \tilde{x}_{3}^{2}
$$

Because of the impossible measurement of the variable, $\tilde{x}_{3}$, the variable, $\sigma$, is used as the following:

$\sigma=\theta+\eta\left(\hat{x}_{3}, v_{2}\right)$

$\dot{\sigma}=\dot{\theta}+\frac{\partial \eta}{\partial \hat{x}_{3}} \dot{\hat{x}}_{3}+\frac{\partial \eta}{\partial v_{3}} \dot{v}_{2}$

With substitution of equation (1) and the derivative of equation (7) in equation (24) we have:

$$
\dot{\sigma}=-l_{1} \frac{\partial \eta}{\partial \hat{x}_{3}}\left(u_{1}-x_{3}\right)+\frac{\partial \eta}{\partial \hat{x}_{3}} \dot{\hat{\xi}}_{1}+\frac{\partial \eta}{\partial v_{2}} \dot{v}_{2}
$$

Since the $v_{2}$ is a turbulence signal is possible to measure, and $x_{3}$ is a state variable and is recognized as the impossible to measure output, we have

$\dot{\hat{\sigma}}=-l_{1} \frac{\partial \eta}{\partial \hat{x}_{3}}\left(u_{1}-\hat{x}_{3}\right)+\frac{\partial \eta}{\partial \hat{x}_{3}} \dot{\hat{\xi}}_{1}+\frac{\partial \eta}{\partial v_{2}} \dot{v}_{2}$

With the conversion of equation (23) to the estimated parameter, $\theta$, we have:

$$
\begin{aligned}
& \hat{\theta}=\hat{\sigma}-\eta\left(\hat{x}_{3}, v_{2}\right) \\
& \tilde{\theta}=\theta-\hat{\theta}=\sigma-\eta-(\hat{\sigma}-\eta) \\
& \tilde{\theta}=\tilde{\sigma} \\
& \dot{\tilde{\theta}}=\dot{\tilde{\sigma}}
\end{aligned}
$$

Therefore we have

$$
\dot{\tilde{\sigma}}=\dot{\sigma}-\dot{\hat{\sigma}}
$$

With substitution of the equations (25) and (26) in equation (31), and substitution of equation (19) and its results in equation (30) the following equation is obtained:

$$
-l_{1} \frac{\partial \eta}{\partial \hat{x}_{3}}=\Gamma \phi
$$

$\eta=\frac{-\Gamma}{l_{1}} \int \phi d \hat{x}_{3}$

Briefly the derived comparison adaptive law is given as following:

$\phi=\left[\begin{array}{c}-\hat{x}_{3}^{2} \\ v_{2}\end{array}\right]$

$\eta=\Gamma\left[\begin{array}{c}\frac{\hat{x}_{3}^{3}}{3 l_{1} a_{1}} \\ \frac{-v_{2} \hat{x}_{3}}{l_{1} a_{1}}\end{array}\right]$

$\frac{\partial \eta}{\partial \hat{x}_{3}}=\Gamma\left[\begin{array}{c}\frac{\hat{x}_{3}^{2}}{l_{1} a_{1}} \\ \frac{-v_{2}}{l_{1} a_{1}}\end{array}\right]$

$\frac{\partial \eta}{\partial v_{2}}=\Gamma\left[\begin{array}{c}0 \\ -\hat{x}_{3} \\ l_{1} a_{1}\end{array}\right]$

\section{THE SIMULATION OF THE OBSERVER WITH MATLAB}

In this part, the simulation of the obtained estimator in part 2

\begin{tabular}{|c|c|c|}
\hline Unit & Value & Parameter \\
\hline$m^{3}$ & 42 & $v_{d}$ \\
\hline bar & 14000 & $\beta_{d}$ \\
\hline$\frac{\mathrm{kg}}{\mathrm{m}^{3}}$ & 0.0121 & $\rho_{d}$ \\
\hline$\frac{\text { bar.s } s^{2}}{m^{6}}$ & 0.16 & $F_{d}$ \\
\hline$\frac{\mathrm{kg}}{\mathrm{m}^{3}}$ & 0.0121 & $\rho_{a}$ \\
\hline$\frac{b a r \cdot s^{2}}{m^{6}}$ & 0.003 & $F_{a}$ \\
\hline$m$ & 1825 & $h_{b i t}$ \\
\hline & {$\left[\begin{array}{cc}10^{-9} & 0 \\
0 & 10^{-9}\end{array}\right]$} & $\Gamma$ \\
\hline & $10^{-2}$ & $l_{1}$ \\
\hline
\end{tabular}
is performed based on the data in [4] and [5] that is obtained from a real wellbore in the MATLAB environment and the Simulink. The estimated results and the variables are compared with the real data. In the specified well, we don't have the formation pressure at $t=3600$ for $10 \mathrm{~min}$ because of the operation of the pipe connection and during that time period, the pressure sensors are inactive.

Table 1 : Required data for the simulation of wellbore for the Grane field of the North Sea.

With integration of the equation (32) we have: 


\section{CONCLUSION}

As mentioned in the introduction, one of the problems during the pipe connection is the lack of monitoring and observation of the formation pressure caused by the pressure sensors installed on the drill bit being out of the wells during the pipe connection. Therefore, it is necessary to utilize adaptive estimators in order to estimate the formation pressure during drilling operations, which enables it to monitor the formation pressure at all times during the operation; not only during the drilling formation but the pipe connection as well. One of the reasons for the monitoring of the formation pressure is to know when the kick off and the blow out happens in the wellbore because, in this situation, the pressure in the wellbore changes suddenly. With knowledge of these pressure changes, it is possible to perform some actions in order to recognize the kick off and to neutralize it.

In part 2 of this paper, the appropriate model is presented for the estimated observer, and in part 3 the base on the presented model, the comparison estimator and the observer are obtained. Finally, in part 4 , the estimator in the MATLAB environment and Simulink is applied to utilize the data in [4], and [5] is obtained from a well located in the Grane field in the North Sea.

The data acquisition is observed in figures 2,3 , and 4. As seen in the figures, at the time, $t=3600$ s, because of the pipe connection operation for $10 \mathrm{~min}$, the mud pump is turned down and the pipes and drilling bit are transferred to the surface. During those $10 \mathrm{~min}$, the formation pressure is obtained by the comparison estimator. According to the figure 3 , after 1 hour and $10 \mathrm{~min}$, the formation pressure obtained from the sensors installed on the bit becomes equal to the pressure of the compression estimator. And the error yielded from the difference of pressure between the estimator and the sensors become zero.

According to the data acquisition, it can be concluded that the estimator can be used in real systems and drilling rigs because of rapid reaction in approaching of the estimated pressure to the real pressure.

\section{REFERENCE}

[1] G .O . Kassa ,2007,"A Simple Dynamic Model of Drilling “,Technical Report ,Statoil , Norway.

[2] H .K. Khalil ,2002,"Nonlinear Systems” , Englewood Cliffs

[3] E .Jahnshahi , K. Salahshoor , R. Kharrat ,2007,"A Modified Distributed Delay Model for Void Wave Dynamics in Gas Lift Oil Wells" ,JPST.

[4] J. Zhou, O . N. Stamnes ,O.M. Aamo ,G.O. Kassa ,2010 "Pressure Regulation with Kick Attenuation in a Managed Pressure Drilling System," The $48^{\text {th }}$ IEEE Conference in Decision and Control ,Shanghai ,P.R. China.

[5] G. Nygaard ,2006 "Multivariable Process Control in High Temperature and High Pressure Environment Using Non-Intrusive Multi Sensor Data Fusion", Phd Thesis, NTNU.

[6] D. Hargreaves, S. Jardine , and B. Jeffryes ,2001 "Early kick detection for deepwater drilling: New probabilistic methods applied in SPE Annual Technical Conference and Exhibition.

[7] H. Santos, E. Catak, J. Kinder, and P. Sonnemann ,2007, "Kick detection and control in oil-based mud: Real welltest results using micro control equipment", in SPE/IADC Drilling Conference, Proceedings, vol. 1. Society of Petroleum Engineers (SPE), Richardson, United States, pp. 429-438.

[8] C. L. Helio Santos and S. Shayegi ,2003, “Micro- ux control :the next generation in drilling process for ultradeepwater", in SPE Latin American and Caribbean Petroleum Engineering Conference, ser. SPE8113, Portof-Spain, Trinildad, West Indies.

[9] M. Doria and C. Morooka , 1997, "Kick detection in coating drilling rigs, in SPE/IADC 39004. IADC/SPE Drilling Conference, Amsterdam.

[10] J .J. Azar and G.R.Samuel,2007,” Drilling Engineering”. Penwell Corporation.

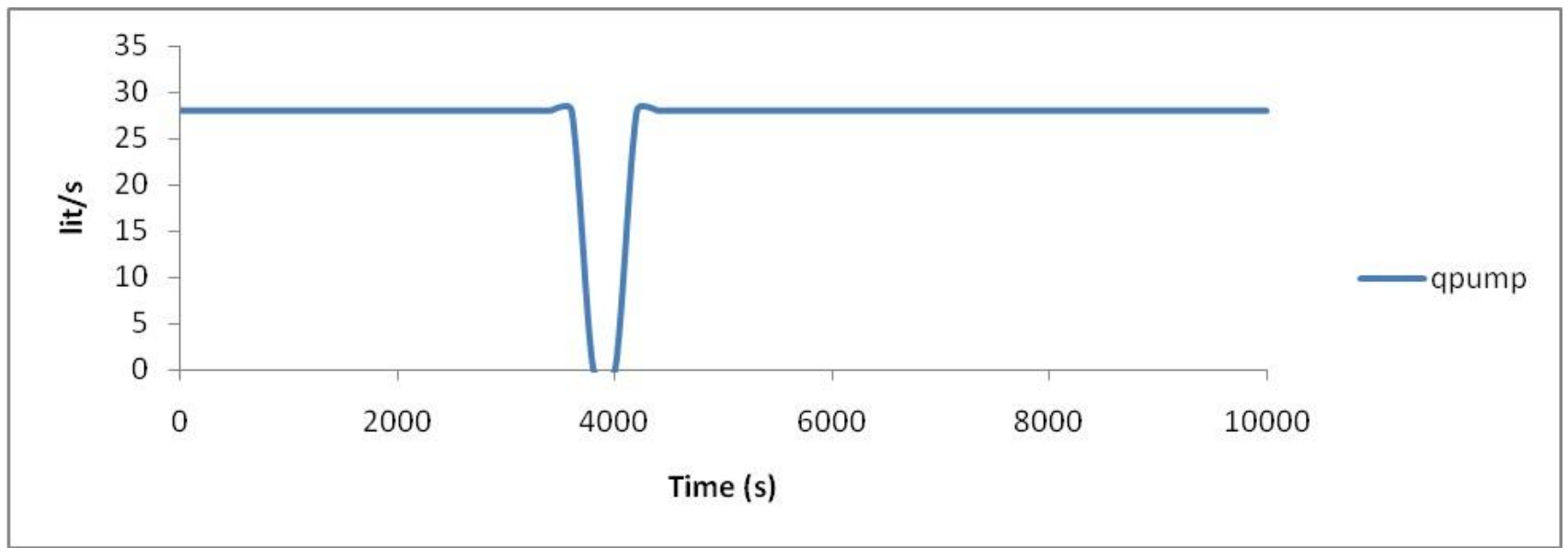

Figure 2.The flow rate of the input drilling mud to the drillstring via mud pump, Grane field of the North Sea 


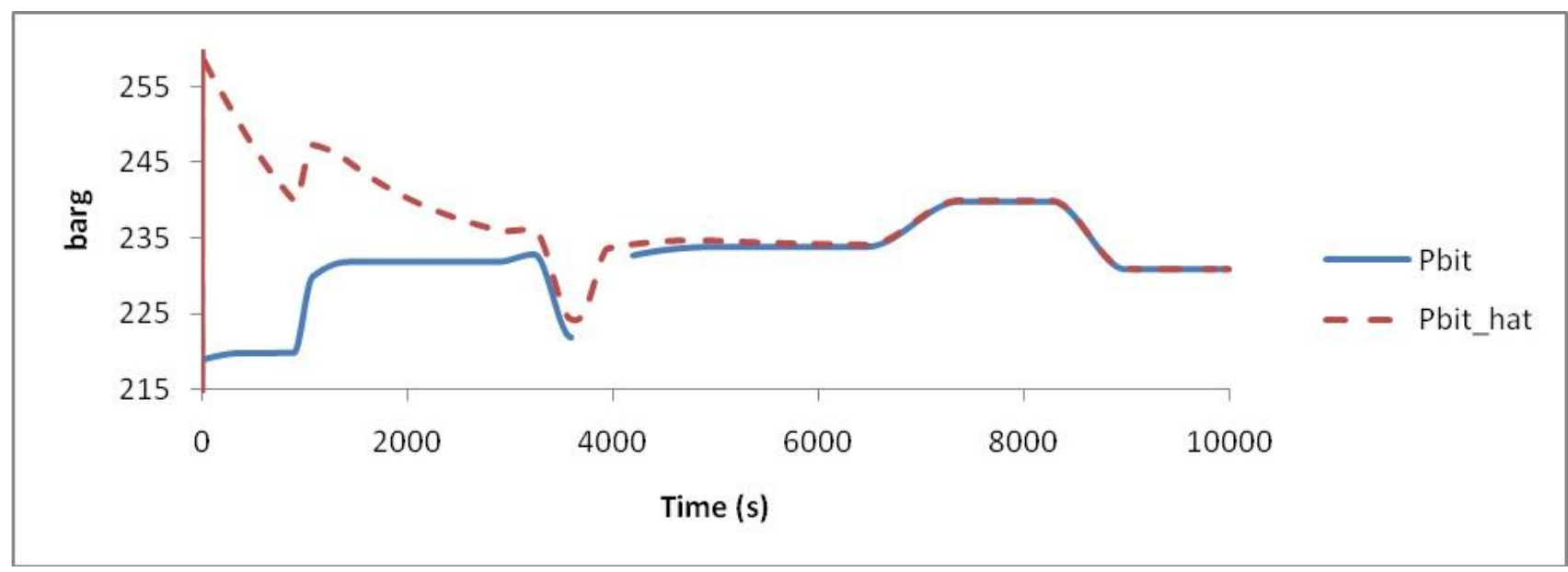

Figure 3.The pressure of the drilling mud at the bottom of the wellbore: 1 - obtained from the pressure sensors on the drilling bit, Grane field located in the North Sea, 2- The estimation of the formation pressure by the comparison estimator.

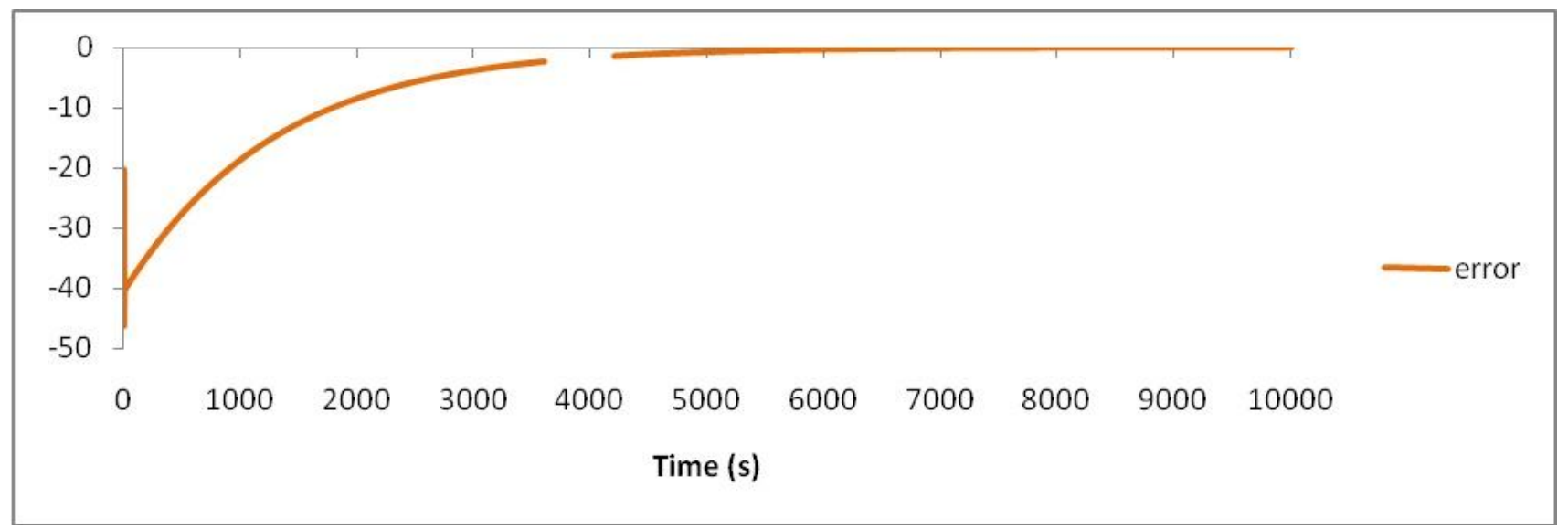

Figure 4.The error yielded from the difference of the sensors pressures and the estimated pressure. 